\title{
Multiple Frequency Solidly Mounted BAW Filters
}

\author{
Alexandre Reinhardt, \\ Jean-Baptiste David, Christine Fuchs \\ CEA LETI, Minatec Campus, \\ Grenoble, France \\ E-mail: alexandre.reinhardt@cea.fr
}

\author{
Marta Clement, Jimena Olivares, \\ Enrique Iborra \\ Grupo de Microsistemas \\ y Materiales Electrónicos, \\ Universidad Politécnica de Madrid \\ Madrid, Spain
}

\author{
Nick Rimmer, Steve Burgess \\ SPP Process Technology Systems UK Ltd., \\ (formerly Aviza Technology) \\ Newport, South Wales, \\ United Kingdom
}

\begin{abstract}
This paper reports the simultaneous fabrication of Receive and Transmit Bulk Acoustic Wave filters for the WCDMA standard on the same die. Both filters are based on Solidly Mounted Resonators using a common Bragg mirror, but with each having a specific piezoelectric film thickness. Electrical measurements reveal that the process steps required to provide the two different piezoelectric film thicknesses on the same die does not impact the electrical performances of resonators and filters and that this approach could thus be generalised to more than two filters.
\end{abstract}

\section{INTRODUCTION}

Acoustic filters are an enabling technology for radiofrequency front-ends, where they are used for RF band pass filtering. Among them, Bulk Acoustic Wave (BAW) filters are commercially available for almost 10 years now, and find their use for stringent filtering applications, where low losses, steep skirts and sharp roll-off are required [1]. They consist in a piezoelectric thin film sandwiched between two electrodes, and operating on a thickness-extensional vibration mode. Therefore, their operation frequency is fully defined by the set of layers from which they are obtained and especially by thicknesses. Unlike other technologies, like Surface Acoustic Wave (SAW), Lamb Wave Resonators, or other Micro Electro Mechanical (MEMS) resonators, their characteristics depend only weakly on their geometric design. This means that a unique set of resonators, operating at a given frequency, can be fabricated at a time. Local addition of mass by depositing an added layer over some resonators enables to shift their frequency through the mass loading effect, and thus enables to obtain the two sets of resonators required to obtain a conventional ladder or lattice type filter. However, it is hardly possible to synthesize two filters for different standards. Instead, only one bulk acoustic wave filter is usually fabricated at a time and current RF front-ends thus require as many filter dies as they include filters. This will surely become a critical problem in mobile phone applications with newer standards making use of even more frequency bands, what will cause an increase in the number of filters necessary in a radiofrequency front-end. Therefore, the integration of these components in systems will become critical.

Previous studies have focused on co-integrating two RF filters on the same die, at least to provide both Receive $(\mathrm{Rx})$ and Transmit (Tx) filters of the same standard on the same die, thus enabling to assemble only a single filter die on a duplexer module. This means being able to provide four sets of resonators having each a different set of resonance frequencies. The most intuitive way is to exploit the massloading effect in a way similar as to the one in which two sets of frequencies are defined in a conventional filter [2]. It has been recently applied for example to the fabrication of a Film Bulk Acoustic Resonator (FBAR) based duplexer for the WCDMA standard [3]. By using both a so-called band tuning layer to define resonators at Rx frequencies, and then a second loading layer to define the parallel resonators of both filters, a set of four frequencies was obtained. The same principle could be similarly applied with Solidly Mounted Resonators (SMR). The advantage of this solution is that it requires only an additional layer and an additional mask level used to pattern the band tuning layer compared to a conventional BAW process. However, this additional band-tuning layer normally causes a reduction of the effective electromechanical coupling factor of resonators, making the synthesis of wide bandwidth filters more difficult. This reduction increases as the frequency shifts between the two filter bands becomes large. This means in practice that this approach can only be used where a reduction in effective electromechanical coupling factor for one of the two filters is acceptable. This also means that this solution is only applicable to two close frequency bands.

In order to fabricate simultaneously filters with arbitrary bandwidths and frequency spacing, without sacrificing effective electromechanical coupling factor, i.e., ultimately, insertion losses and ripple, several piezoelectric layer thicknesses need to be provided on the same substrate [4]. A first approach proposed to implement such a device was first to deposit the thinner piezoelectric film, then to protect it where the highest frequency filter will be located, and finally to deposit an additional thickness of the piezoelectric layer where the lowest frequency filter will be located [5]. However, authors have shown that in practice, with Aluminum Nitride (AIN), piezoelectric properties of the thicker film are decreased compared to the initially deposited one. Investigations have proven that the cause of this decreased piezoelectric properties is attributed to either a bad crystallinity of the re-grown layer, or by a regrowth of AlN with a reverse polarity, causing a partial cancellation of the effective piezoelectricity of the piezoelectric film. The origin of this phenomenon is the surface 
degradation of the initial AlN film, caused by reaction to chemical agents during photolithography steps, but it was also noticed, although less significantly, in samples having only been exposed to air for some time. The conclusion of this first approach was that oxidation or reaction with humidity may trigger surface deterioration and cause re-growth with the wrong polarity.

Another approach would be to start with the deposition of the thickest piezoelectric layer first, and then to partially etch this film to provide a thinner layer where the highest frequency filter will be located. The advantage is that it ensures that the structural quality of AlN remains unaffected. However, there are two critical issues: first AlN is still exposed to chemicals during photoresist development or stripping, which are known to damage it, in partially etching it and increasing its surface roughness. Conjugated effects of roughness increase and surface contamination of the piezoelectric film are expected to prove detrimental towards quality or effective electromechanical coupling factors of resonators. Secondly, although the deposition processes of AlN in an industrial environment is very well controlled and reaches unmatched thickness homogeneity in order to reduce frequency dispersions, while offering growth rates compatible with mass production in an industrial line, etching processes are much less optimized. Processes with reasonable etch rates suffer from much lower uniformity and thickness control, especially when it comes to partially etch a layer without proper etch-stop. On the other hand, some equipments, for example dedicated to frequency trimming of resonators, offer an excellent control over etching depth and uniformity, but at the expense of very slow etch rates, making these approaches difficult to implement for mass production. So using direct partial etching of AlN does also not seem to be an interesting alternative.

To avoid the drawbacks of the above-mentioned methods for fabricating several BAW filters simultaneously, we propose in this paper a new approach based on multiple depositions and patterning of the piezoelectric layer [6]. In section II, we discuss the design of two filters compatible with the proposed approach. Then, in section III, we describe the fabrication of both Reception and Transmission filters for the WCDMA standard. Section IV provides the electrical characteristics of the fabricated filters and a comparison with conventional filters with the same design.

\section{WCDMA DUPLEXER FILTERS DESIGN}

The two filters were designed to match the WCDMA standard, using the procedure described in [7], [8]. Based on a first estimation of achievable resonator electromechanical coupling and quality factors, Butterworth-Van Dyke (BVD) lumped element models of resonators were built and used for filter synthesis. An equivalent filter model, based on an initial filter architecture, is first proposed. The transmission filter is based on a $50 \Omega$ single stage ladder topology, with only three resonators, since most of the rejection will be provided by the duplexer itself. The reception filter is implemented as a double lattice topology, as it requires higher rejection and

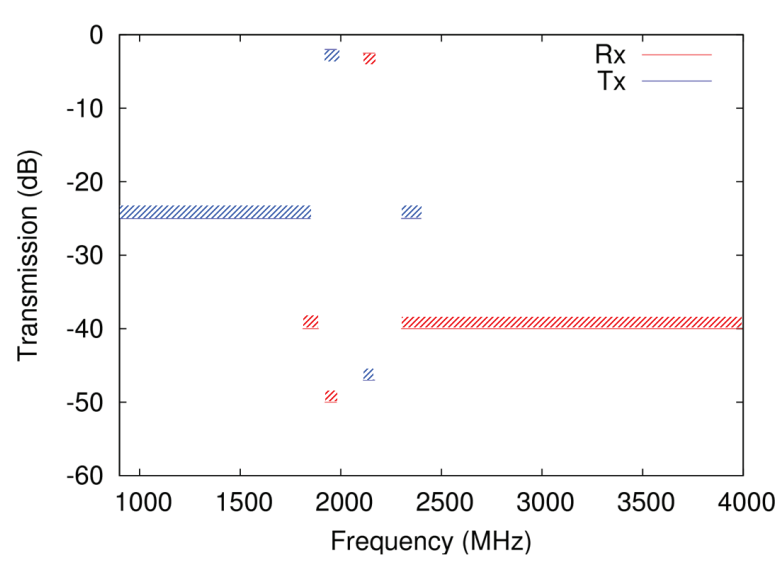

Fig. 1. Filter masks for the WCDMA duplexer : antenna to Rx path (red) and Tx to antenna path (blue).

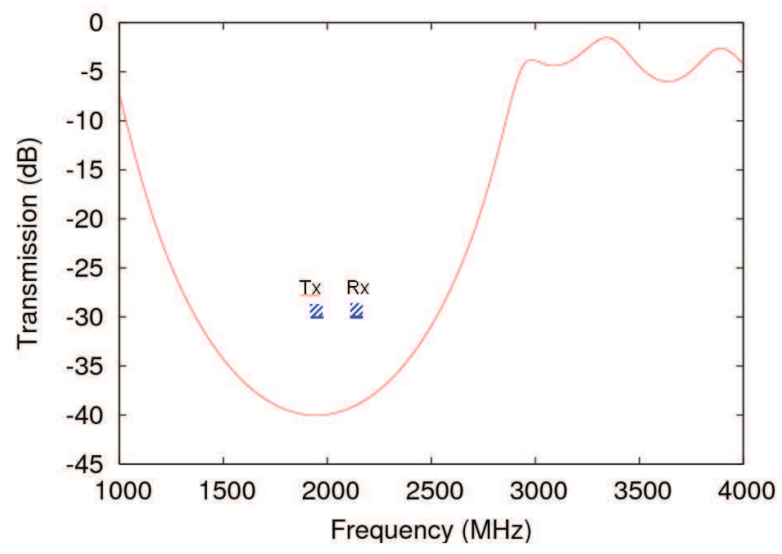

Fig. 2. Transmission coefficient of the designed SiN/SiOC Bragg mirror.

differential input and output. This model is then optimized to meet specifications for the whole duplexer module, which are summarized by the masks shown in Figure 1. The output of this optimization basically provides required resonator static capacitances and, more importantly for this study, resonance and antiresonance frequencies.

From this basis, a more precise Mason model of resonators is used. First, a Bragg mirror is designed to cover the two filter bands. This mirror is made of $\mathrm{SiN}$ and $\mathrm{SiOC}$, which exhibit an acoustic impedance ratio larger than 6 and thus can provide a mirror offering a transmission lower than $-30 \mathrm{~dB}$ over a bandwidth of more than $1 \mathrm{GHz}$ with 3 pairs of layers, as seen in Figure 2. Such a wide bandwidth is more than sufficient to cover the two filter bands. It also ensures a rather small change in reflection coefficient between the filters: this is mandatory to ensure that both devices will benefit from a quasi-similar acoustic isolation and from small changes in phase of the reflection coefficient, what is necessary for the maximum achievable effective electromechanical coupling factor of resonators to remain similar for the two filters.

Two Molybdenum/AlN/Molybdenum piezoelectric stacks have been designed so that resonators simultaneously reach 
the specified resonance and antiresonance frequencies in the series paths of the two filters. This design also takes into account an additional $\mathrm{SiN}$ passivation layer whose thickness is otherwise defined for reliability reasons [9]. However, we added the additional constraint to try to have as many common layers as possible, to ease subsequent processing. For the set of frequencies defined during the optimization of the filter topology, it proved impossible to achieve sufficient electromechanical coupling factor with the same piezoelectric layer thicknesses or with the same bottom or top electrodes. However, the required amount of mass loading necessary to induce frequency shifts of 69 and $56 \mathrm{MHz}$ between series and parallel resonators, respectively for the $\mathrm{Rx}$ and $\mathrm{Tx}$ filter, is obtained by almost adding the same thickness for the two material stacks. This means that the same mass-loading can be applied at least to the two filters.

\section{FILTER FABRICATION}

From these acoustic design considerations, one can see that the fabrication of the two filters differs only at the level of the two electrodes and at the level of the piezoelectric film. Therefore, for most of the fabrication process, we use a conventional BAW fabrication process. We start with the deposition onto a high resistivity wafer of the 7 layers forming the common Bragg mirror, alternatively SiOC (335 nm) and SiN (1200 nm). These layers are deposited by chemical vapor deposition (CVD). The whole deposition process has been optimized to minimize stress wile providing a flat and smooth surface on which to grow the piezoelectric stacks.

A Molybdenum film is deposited on top of these Bragg mirrors by sputtering the thickest of the two bottom electrodes, i.e. $400 \mathrm{~nm}$ of Molybdenum corresponding to the thickness required for the Tx filter. This metal film is then locally partially etched over $50 \mathrm{~nm}$ to reach the thickness required by the Rx filter. To ensure a precise control of this thickness difference, we use very slow etching process in an ion beam tool. Then, the bottom electrodes for the two filters are simultaneously patterned using standard photolithography and a fluorine-based dry etching step.

A first piezoelectric AlN film close to $1300 \mathrm{~nm}$ is deposited by reactive pulsed DC sputtering, followed by the deposition of the first top molybdenum electrode $(140 \mathrm{~nm})$ in the same cluster tool (a Sigma Fxp sputtering system from Aviza Technologies) to avoid any vacuum break. These layers form the piezoelectric stack for the Tx filter. Large patterns are then defined on the top metallization to define areas where Tx filters will reside, using standard photolithography and dry etching. The molybdenum film then serves as a metal mask for the chemical wet etching of AlN. We checked that this chemical etching leaves a flat surface without any AIN residues which could locally degrade the growth of the second piezoelectric stack.

This second piezoelectric stack is deposited the same way as the previous one on top of the existing materials. It consists in an AlN film of $1150 \mathrm{~nm}$ and a Mo film of $120 \mathrm{~nm}$. The top Mo film is patterned to define a second metal mask,

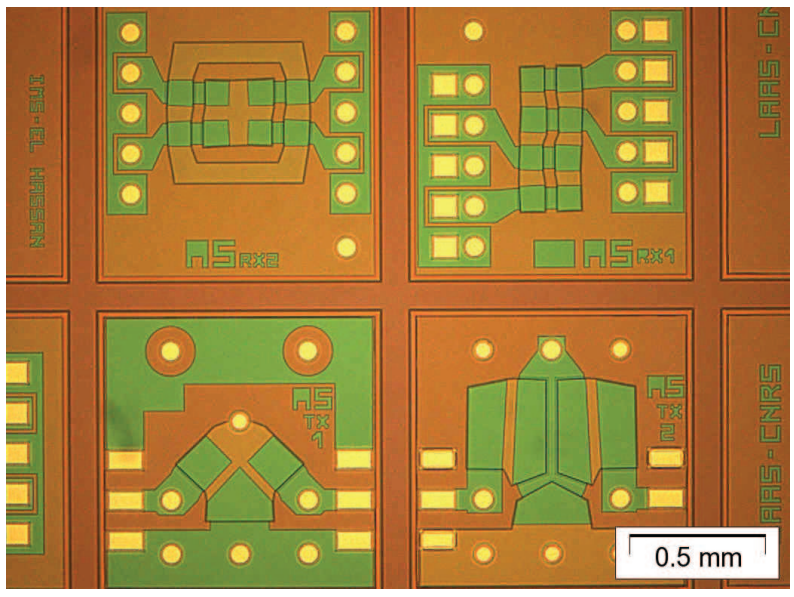

Fig. 3. Optical photograph of Rx and Tx filters after fabrication.
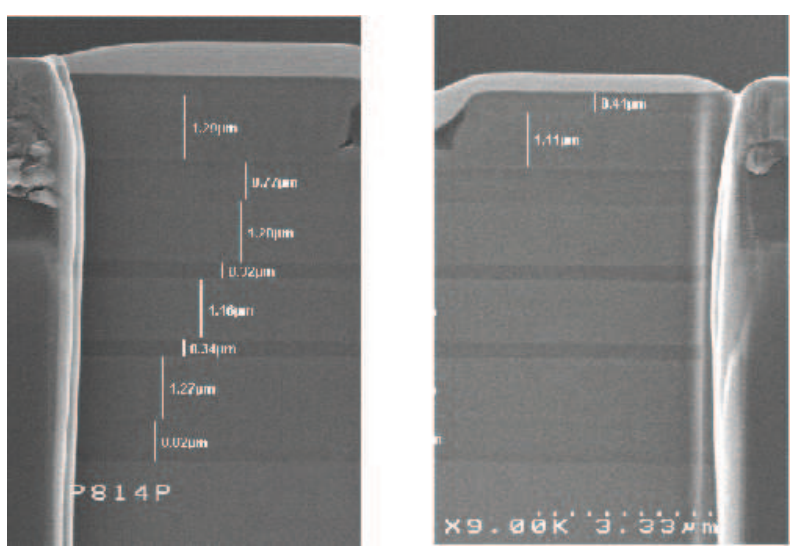

Fig. 4. SEM cross sections of Tx (left) and Rx (right) islands.

complementary of the previous one, which defines the areas where the Rx filters will reside, and the second AlN layer is etched again. At this stage, the first Mo metal mask protects the first AlN film, acting as an etch stop for the second AlN, so that two kinds of islands with varying piezoelectric stacks are defined. Microscope inspection revealed that no remains of the second AlN film were noticed over the top electrode of Tx islands after this etching step, so that the fabrication process could be continued safely. We also assessed the crystalline quality of AlN films by rocking curve measurements. No noticeable change in AlN texture was noticed between Rx and Tx islands and between reference wafers which have only seen the deposition of one piezoelectric stack.

Frequency separation between series and parallel resonators for the two filters is obtained by partially etching the two top molybdenum layers over the locations where series resonators will reside. As for the bottom electrode, we partially etch these layers using slow ion beam etching for a precise thickness control and a high on-wafer uniformity. The top electrodes are then patterned and vias are etched in the AlN islands using photolithography and another wet etching to reach the bottom electrodes. Devices are then passivated using an SiN film covering the whole surface, except where contacts towards 


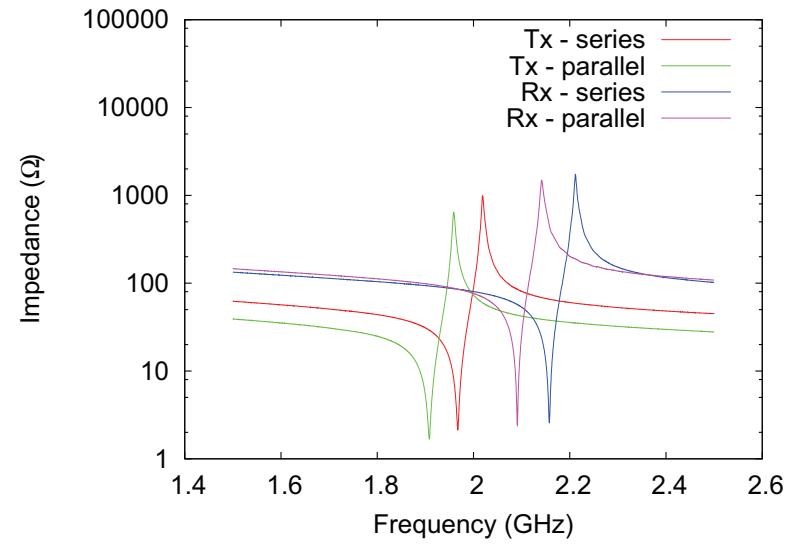

Fig. 5. Electrical response of $\mathrm{Tx}$ and $\mathrm{Rx}$ resonators measured on the same wafer die.

top and bottom electrodes are opened by fluorine based dryetching. Finally, a thick aluminum layer is deposited and patterned to cover vias and to form contact pads for RF probing.

Figure 3 is an optical photograph showing filters after fabrication. Each filter resides on an $1 \times 1 \mathrm{~mm}^{2}$ die having its own piezoelectric stack. Vertical and horizontal lines separate islands corresponding to the $\mathrm{Rx}$ or the Tx material stack. Due to the relatively small change in AlN thickness between the two types of filter, no noticeable colour change can be observed. Figure 4 shows cross sections of Tx and Rx islands obtained on the same wafer at a small distance from each other.

\section{ELECTRICAL CHARACTERIZATIONS}

After fabrication, a set of 167 test resonators regularly distributed accross each wafer were tested using RF probes connected to a vector network analyzer and calibrated using the conventional SOLT method. For each of them, we extracted resonance and antiresonance frequencies, as well as quality and electromechanical coupling factors. Figure 5 shows sample impedance responses of resonators for the $\mathrm{Rx}$ and Tx filters measured on the same wafer die. Figure 6 gives the values of electromechanical coupling factor averaged over each wafer (i.e. over 167 samples) compared to reference wafers having only seen the fabrication of one filter. As can be seen, changes in resonator properties remain within measurement uncertainties, showing that the multiple frequency process has not significantly impacted resonator performances. Measurements of two filters located in the same $2 \mathrm{~mm}^{2}$ die are shown in Figure 7: these two filters exhibit excellent performances: respectively 1 and $2.3 \mathrm{~dB}$ insertion losses for the Transmit and Receive filters. It is to be noted that the somehow reduced rejection of the Transmit filter will be greately improved when mounted inside the duplexer chip.

\section{CONCLUSION}

We have simultaneously fabricated Rx and Tx filters for the WCDMA standard based on SMR. The fabrication process

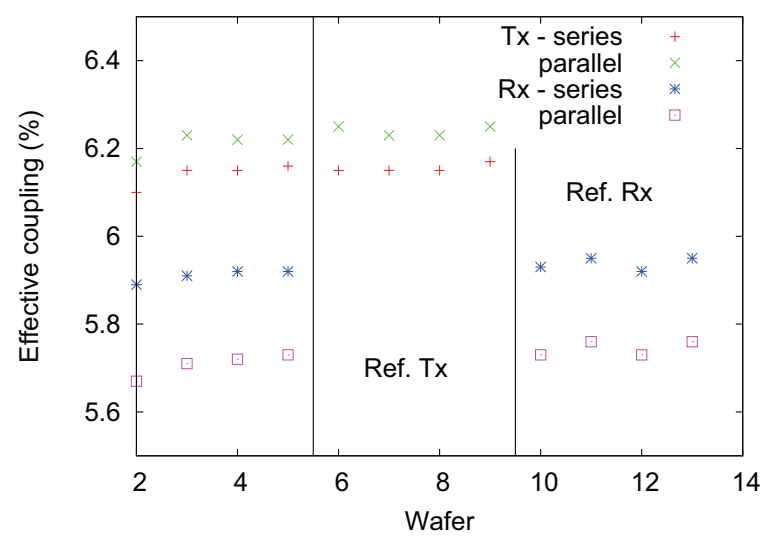

Fig. 6. Comparison of average electromechanical coupling factors measured between wafers having seen the fabrication of the two filters and reference filters.

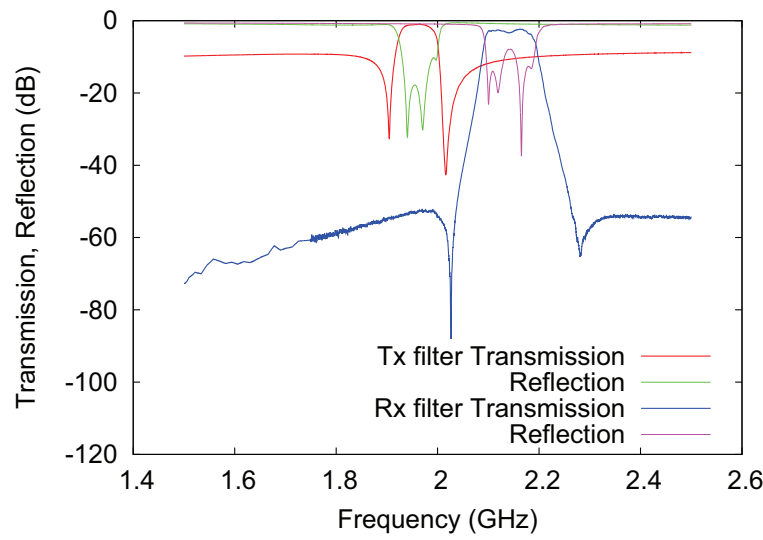

Fig. 7. Transmission and reflection measurement for adjacent $\mathrm{Tx}$ and $\mathrm{Rx}$ filters.

employed makes use of the deposition and patterning of two piezoelectric films which are then patterned thanks to the very large etching selectivity between AIN and the Molybdenum electrodes used. Electrical measurements reveal that no change in performances is induced in individual resonators or in filters compared to a standard single-frequency BAW process, proving the interest of this method, and moreover does not put strict constraints in terms of acoustic design of resonators. Moreover, this approach can be easily generalized to alternative technologies using other electrode materials or other piezoelectric films. It could also be generalized to a larger number of filters, being only limited by the ability to design a common Bragg mirror suitable for all filters.

\section{ACKNOWLEDGMENT}

The authors acknowledge financial support from the European Commission in the FP6 IST 2004-027003 MOBILIS project. They want to thank the whole LETI BAW team for fruitful discussions, for their support and for their deep involvement in device fabrication and characterization. They also thank all partners of the MOBILIS project for their contributions. 


\section{REFERENCES}

[1] R. Aigner, "SAW and BAW technologies for RF filter applications: a review of the relative strengths and weaknesses," in 2008 IEEE International Ultrasonics Symposium Proceedings, 2008, pp. 582-589.

[2] K. M. Lakin, K. T. McCarron, J. Belsick, and R. Rose, "Filter banks implemented with integrated thin film resonators," in Proceedings of the 2000 IEEE International Ultrasonics Symposium, 2000, pp. 851-854.

[3] K. Nam, Y. Park, B. Ha, C. Kim, J. Shin, S. Yun, J. Pak, G. Park, and I. Song, "Monolithic 1-chip FBAR duplexer for W-CDMA handsets,' Sensors and Actuators A, vol. 143, pp. 162-168, 2008.

[4] J. Ella and H. Pohjonen, "BAW filters having different center frequencies on a single substrate and a method for providing the same," United States Patent US 6,518,860 B2.

[5] F. Martin, P. Muralt, M. Cantoni, and M. Dubois, "Re-growth of c-axis oriented AlN thin films," in Proceedings of the 2004 IEEE International Ultrasonics Symposium, 2004, pp. 169-172.
[6] E. Iborra, M. Clement, and J. Olivares, "Device for filtering bulk acoustic waves," Patent application WO2010/116011 A1, Oct. 2010.

[7] S. Giraud, S. Bila, M. Chatras, D. Cros, and M. Aubourg, "Bulk acoustic wave filter synthesis and optimization for UMTS applications," in Proceedings of the 39th European Microwave Conference, 2009, pp. 456-459.

[8] M. Clement, E. Iborra, J. Olivares, N. Rimmer, S. Giraud, S. Bila, and A. Reinhardt, "DCS Tx filters using AlN resonators with iridium electrodes," IEEE Transactions on Ultrasonics, Ferroelectrics and Frequency Control, vol. 57, no. 3, pp. 518-523, March 2010.

[9] R.-Y. Fillit, B. Ivira, J. Boussey, R. Fortunier, and P. Ancey, "Structural and thermal investigation for FBAR reliability in wireless applications," in Proceedings of the 43rd Annual International Reliability Physics Symposium, 2005, pp. 342-346. 